\title{
Dos contextos da prática à (co)construção do modelo de cuidados de enfermagem de família*
}

\author{
FROM PRACTICE CONTEXTS TOWARDS THE (CO)CONSTRUCTION OF FAMILY \\ NURSING CARE MODELS
}

\author{
DE LOS CONTEXTOS DE LA PRÁCTICA A LA (CO)CONSTRUCCIÓN DEL MODELO \\ DE CUIDADOS DE ENFERMERÍA DE LA FAMILIA
}

Maria Henriqueta de Jesus Silva Figueiredo', Maria Manuela Ferreira Pereira da Silva Martins ${ }^{2}$

\begin{abstract}
RESUMO
Face aos desafios que se colocam aos enfermeiros, no desenvolvimento de cuidados centrados na família como contexto e unidade de intervenção, realizamos uma pesquisa direcionada para a intervenção familiar, no contexto dos Cuidados de Saúde Primários. De natureza qualitativa, adotamos a metodologia de investigação-ação, numa trajetória de cinco etapas, integradas num ciclo de estudos. Dos resultados do diagnóstico, através da análise comparativa dos modelos conceituais com as práticas de cuidados à família, emergiu a necessidade da mudança iniciada com um processo formativo. Tendo como referenciais o Modelo Sistêmico, o Modelo Calgary de Avaliação da Família e o Modelo Calgary de Intervenção na Família, reconstruiu-se o modelo de cuidados de enfermagem à família, operacionalizando-o através da definição das áreas de atenção e intervenção. A co-construção do modelo a partir das necessidades identificadas pelos enfermeiros, legitimado pelas tomadas de decisão dos mesmos, constituise como base de discussão no contexto da enfermagem de família.
\end{abstract}

\section{DESCRITORES}

Família.

Enfermagem familiar.

Modelos de enfermagem.

\begin{abstract}
As nurses face challenges in the development of care focused on the family as a context and unit of intervention, this research aimed to identify family intervention processes in the context of Primary Healthcare. This qualitative-based study adopted the investigation-action approach on a study plan's five-step path. By means of the comparative analysis of family care practices and conceptual models, the diagnosis results brought forth the need for change triggered by training processes. Having the Systemic Model, the Calgary Family Assessment Model, and the Calgary Family Intervention Model as references, the family nursing care model was (re)constructed and operationalized through the definition of attention and intervention areas. The (re) construction of the model from the needs identified by nurses and legitimated by their decision-making processes is the basis for family nursing focused discussions.
\end{abstract}

\author{
KEY WORDS \\ Family. \\ Family nursing. \\ Models, nursing.
}

\begin{abstract}
RESUMEN
Frente a los desafíos que se colocan a los enfermeros, en el desarrollo de cuidados centrados en la familia, como contexto y unidad de intervención, realizamos una investigación dirigida para la intervención familiar, en el contexto de los Cuidados de Salud Primarios. De naturaleza cualitativa, adoptamos la metodología de investigación acción, en una trayectoria de cinco etapas, integradas en un ciclo de estudios. De los resultados del diagnóstico, a través del análisis comparativo de los modelos conceptuales con las prácticas de cuidados a la familia, surgió la necesidad del cambio, iniciado con un proceso formativo. Teniendo como marco teórico el Modelo Sistémico, el Modelo Calgary de Evaluación de la Familia y el Modelo Calgary de Intervención en la Familia, se (re)construyó el modelo de cuidados de enfermería a la familia haciéndolo operacional a través de la definición de las áreas de atención e intervención. La (co)construcción del modelo a partir de las necesidades identificadas por los enfermeros, legitimado por las tomas de decisión de los mismos, se constituye en la base de discusión, en el contexto de la enfermería de familia.
\end{abstract}

\section{DESCRIPTORES}

Familia.

Enfermería de la familia.

Modelos de enfermería.

\footnotetext{
* Extraído da tese "Enfermagem de família: um contexto do cuidar", Instituto de Ciências Biomédicas Abel Salazar, Universidade do Porto, $2008{ }^{1}$ Enfermeira Especialista em Enfermagem Comunitária. Professora Adjunta da Escola Superior de Enfermagem do Porto. Porto, Portugal. henriqueta@esenf.pt 2 Enfermeira. Professora Coordenadora da Escola Superior de Enfermagem do Porto. Porto, Portugal. mmartins@esenf.pt
} 


\section{INTRODUÇÃO}

Os cuidados à família desenvolveram-se a par da evolução dos cuidados de enfermagem. As enfermeiras prestavam os cuidados nos contextos onde as pessoas estavam inseridas ${ }^{(1)}$, havendo evidência da existência de práticas dirigidas às famílias desde Florence Nightingale, expressas pela preocupação em incluir os membros da família no desenvolvimento dos cuidados $^{(2)}$.

No passado o conceito de família era associado ao núcleo familiar, casal que vivia com os seus filhos biológicos e eventualmente com um dos pais de um dos cônjuges. Actualmente existe uma grande diversidade de tipos e estruturas familiares. Às múltiplas formas de organização familiar estão associadas não só as alterações nos papéis de género, mas também a diversidade de interacções conjugais e os processos de recomposição familiar ${ }^{(3)}$. Na literatura actual encontramos conceitos que incluem as pessoas significativas como membros da família( ${ }^{(4)}$, numa tendência de auto definição cada vez mais evidenciada pela diversidade dos laços que caracterizam a família como grupo ${ }^{(1,2)}$. Os laços estabelecidos entre o grupo familiar, associados aos contextos específicos do seu desenvolvimento, tornam a família um grupo com identidade própria, um sistema aberto de comunicação multidireccional| ${ }^{(5)}$, numa perspectiva multiversa da família.

Para a compreensão da família, como unidade, é essencial que a conceptualizemos através de um paradigma que nos permita entender a sua complexidade, globalidade, diversidade, unicidade, entre muitas outras características inerentes à sua multidimensionalidade e que ultrapasse as definições associadas à consanguinidade e afinidade. Da triangulação entre a Teoria Geral dos Sistemas de Bertanlaffy ${ }^{(6)}$ que definiu as leis gerais que permitem conhecer as características dos sistemas, a Cibernética de Norbert Weiner ${ }^{(7)}$ que estudou a forma de funcionamento dos sistemas e a Teoria da Comunicação Humana de Paul Waltzlawick em colaboração com outros teóricos da Escola de Palo Alto ${ }^{(8)}$, que fizeram a ligação entre os elementos que compõem cada sistema, emergiu o Modelo Sistémico ou Pensamento Sistémico ${ }^{(9)}$. O estudo dos sistemas surgiu da necessidade de amplificar a visão ao todo em vez de decompor as partes, concebendo a unidade familiar como todo e parte indivisível e em que nenhum dos dois termos pode ser redutível ao outro ${ }^{(6)}$. Neste contexto o Modelo Sistémico enfatiza o sistema de relações e interacções intrínsecas à vida da família, colocando o foco na complexidade do contexto.

A enfermagem de família surge como arte e ciência(2), alicerçada ao pensamento sistémico com uma abordagem que ultrapassa o paradigma da ciência tradicional, permitindo a compreensão de todos os factores que inteiram a unidade familiar.
Os pressupostos associados à enfermagem de família integram uma filosofia de parceria com a família, visando apoiála na procura de soluções para os problemas identificados, englobando-a no planeamento e execução das intervenções ${ }^{(1,10)}$. Direccionada a todos os tipos de famílias, não existindo famílias impróprias ou inadequadas ${ }^{(11)}$, centra-se nos pontes fortes dos membros da família e do grupo familiar promovendo o seu crescimento e desenvolvimento. Emerge da mutualidade entre saúde e funcionamento da família ${ }^{(12)}$, sendo que os problemas de saúde influenciam as percepções e comportamentos da família, do mesmo modo que as percepções e comportamentos desta influenciam a saúde dos seus membros. A família constitui-se como factor de adversidade ou de protecção relativamente aos processos de saúde e doença dos seus membros e aos processos de adaptação inerentes ao seu desenvolvimento. Os processos de adaptação a novas situações, sejam elas internas ou externas, a fim de assegurar a mudança e continuidade da família, serão, pois, sempre revestidos de mais ou menos stress ${ }^{(13)}$.

A partir destes pressupostos, que representam alguns dos aspectos mais importantes caracterizadores do sistema familiar, compreendemos o enfoque da enfermagem de família nas relações e nas interacções, em que a finalidade da intervenção é a mudança, numa perspectiva dinâmica de bem estar nas dimensões interaccionais, funcionais e estruturais do sistema familiar. Em síntese, os objectivos da enfermagem de família centram-se na capacitação da família no desenvolvimento de competências que permitam uma vivência saudavel dos seus constantes processos de mudança. Os cuidados de enfermagem desenvolvem-se ao longo do ciclo vital da família, nas situações de saúde e doença, com enfase nas respostas da família aos problemas reais ou potenciais ${ }^{(1)}$.

É neste contexto de cuidados, baseados numa abordagem sistémica, que a família é compreendida através da sua complexidade e unicidade. E cada uma das partes, os indivíduos que integram o sistema, é percepcionada com uma estrutura própria, dinâmica, com uma história única, compreendida na interação contextual ${ }^{(14)}$.

A avaliação e intervenção de enfermagem exigem a utilização de modelos que permitam a concepção de cuidados orientados tanto para a colheita de dados como para o planeamento das intervenções. Nesta perspectiva o Modelo Calgary de Avaliação da Família (MCAF) ${ }^{(1)}$ com uma perspectiva multiversa do sistema família, apresenta-se com uma estrutura multidimensional, que engloba três dimensões: estrutural, de desenvolvimento e funcional. Cada uma destas dimensões integra várias categorias e sub-categorias que, no seu conjunto, permitem a avaliação sistémica da família, numa perspectiva dinâmica e de continuidade.

A avaliação estrutural(1) integra aspectos do conhecimento da estrutura da família, integrando aspectos relati- 
vos à sua estrutura interna, externa e de contexto. Dá-nos uma visão sobre a composição da família, o vínculo entre os seus membros e entre estes e outros elementos significativos como a família alargada ou os sistemas mais amplos, assim como o contexto onde a família se insere.

A avaliação de desenvolvimento( ${ }^{(1)}$ possibilita a compreensão dos fenómenos exclusivos da família, associados ao seu crescimento, à sua trajectória evolutiva a nível funcional, estrutural e interaccional. O conhecimento desta trajectória, articulada com as transformações na organização familiar decorrentes do ciclo de vital, possibilita o desenvolvimento de cuidados antecipatórios para capacitar a família na efectivação das suas tarefas desenvolvimentais.

A avaliação Funcional(1) ${ }^{(1)}$ caracteriza a forma como os membros da família interagem uns com os outros. O Funcionamento Instrumental centra-se nas actividades do quotidiano da vida da família, enquanto que o Funcionamento Expressivo direcciona-se para os padrões de interação, subdividido em nove subcategorias: comunicação emocional; comunicação verbal; comunicação não verbal; comunicação circular; solução de problemas; papéis; influência e poder; crenças; alianças e uniões. Estes processos de interação parecem ter maior efeito sobre o estado de saúde da família do que a sua estrutura ou função(2).

A dinamicidade e inter penetrabilidade das dimensões, possibilita o planeamento de intervenções, com a família, visando dar resposta aos problemas identificados, através da promoção da mudança num ou em vários domínios do funcionamento da família: cognitivo, afectivo e comportamental ${ }^{(1)}$. Julgamos assim que a utilização do $\mathrm{MCAF}^{(1)}$ permite aos enfermeiros proporem intervenções que dêem resposta às necessidades das famílias em cuidados, identificando, com rigor, tais necessidades.

Identificar algumas áreas de intervenção na família em que haja ganhos em saúde sensíveis aos cuidados de enfermagem, de forma a conseguir definir as áreas de atenção e implementar estratégias conducentes à produção de resultados, pensamos ser uma etapa para a construção do corpo de conhecimentos específicos na área da enfermagem de família.

\section{OBJECTIVOS}

Neste contexto, propusemo-nos desenvolver uma pesquisa que possa contribuir para melhorar os cuidados de enfermagem prestados às famílias, no contexto comunitário, sendo definidos os seguintes objectivos: compreender a intervenção familiar dos enfermeiros de Cuidados de Saúde Primários; contribuir para a definição do corpo de conhecimentos de enfermagem na área de enfermagem de família.

\section{MÉTODO}

A pesquisa é de natureza qualitativa, utilizando-se a metodologia de investigação acção, visando a implementação de novas práticas nos cuidados à família através de um conhecimento prático e o desenvolvimento da teoria ${ }^{(15)}$. Inerente à metodologia, seguimos uma trajectória flexível que adequamos à complexidade das interacções do contexto onde se pretendeu a produção de novos percursos da prática com as famílias. Assim, integrando um ciclo de estudos, definimos cinco fases diferenciadas, conducentes à prossecução dos objectivos da pesquisa e consideradas necessárias para a definição compreensiva da Investigação Acção: Diagnóstico; Planeamento da Acção; Implementação; Avaliação das Mudanças da Prática; Identificação do Inquirido.

A população da pesquisa foi constituída pelos enfermeiros de família de um Centro de Saúde do distrito do Porto, no norte de Portugal, que apresentava as seguintes características: metodologia organizacional de Enfermeiro de Família; utilização do Sistema de Apoio à Prática de Enfermagem (SAPE), que permite a informatização dos registos de enfermagem com a possibilidade de analisar a documentação produzida pelos enfermeiros.

Para o desenvolvimento das várias fases do ciclo de estudo, iniciado em Janeiro de 2005, utilizamos triangulação metodológica, com a aplicação de questionários, análise de documentação, debates de grupo e notas de campo.

Foi obtida a concordância de todos os enfermeiros em participarem na pesquisa, após a aprovação do Projecto de Investigação pela Comissão Ética da Instituição onde se realizou, (Oficio № 4126/CA/AA).

\section{RESULTADOS E DISCUSSÃO}

Apresentamos os resultados e respectiva discussão de acordo com a trajectória metodológica adoptada. Na fase de diagnóstico, cujos resultados apresentamos a seguir, realizamos três estudos, que nos permitiram identificar de forma global os problemas do contexto da prática, no âmbito dos cuidados direccionados às famílias. São discutidos os resultados de cada um dos estudos realizados nesta fase e feita uma análise global que permitiu a continuidade da pesquisa através do planeamento da acção e da implementação das estratégias de mudança.

\section{Fase de diagnóstico}

Nesta fase do ciclo de estudos pretendemos realizar uma análise comparativa entre os modelos expostos e os modelos em uso relativamente às práticas de cuidados de enfermagem centradas na família como unidade de cuidados. Definimos modelos expostos como os modelos conceptuais definidores da enfermagem de família, com a integração dos modelos formativos; sendo que os modelos em uso correspondem aos aspectos decisórios das práticas, os elementos estruturantes das mesmas e os seus significados ${ }^{(16)}$.

Para a identificação e análise dos modelos expostos, além da pesquisa exaustiva sobre as teorias e modelos definidores da enfermagem de família, analisamos os modelos formativos de enfermagem de família através da análi- 
se do plano de estudos do Curso de Licenciatura em Enfermagem (CLE), das instituições de ensino de enfermagem do distrito do Porto, ao que designamos por Estudo 1.

\section{Estudo 1 - Os cuidados de enfermagem à Família no Plano} de Estudos no CLE

Com este primeiro estudo pretendemos identificar as unidades curriculares no Plano de Estudos do CLE que integrem no seu planeamento temáticas no âmbito da enfermagem de família e analisar os objectivos, conteúdos e bibliografia expressos nas unidades curriculares identificadas.

O estudo é descritivo e de natureza qualitativa. Foram consideradas cinco instituições que ministram o CLE, implantadas no Norte do País, sendo três delas públicas e duas privadas.

A recolha de dados foi feita através de análise documental do Plano Curricular do CLE, utilizando-se a informação cedida pelas instituições e/ou a informação disponibilizada nos sites das respectivas instituições, no decorrer dos meses de Março, Abril e Maio de 2005.

O tratamento e análise dos dados foram realizados por análise de conteúdo das unidades curriculares com expressão de uma ou mais unidades de análise.

Verificou-se uma grande heterogeneidade nos Planos de Estudos das instituições, quer nas características gerais dos Planos, quer na estruturação dos conteúdos sobre Família e a sua distribuição pelas diferentes unidades curriculares.

Os conteúdos expressos integram cinco categorias: conceitos e tendências da família; modelos e teorias de enfermagem de família; teorias das ciências sociais da família; processos de saúde/doença; família como alvo dos cuidados.

A análise conjunta das cinco instituições sugere que os objectivos e conteúdos expressos são conducentes ao desenvolvimento de competências para cuidar a família, contudo a fragmentação dos mesmos por instituição, consideramos como elemento dificultador da integralidade do conhecimento.

Para a identificação e análise dos modelos em uso, desenvolvemos dois estudos, designados como Estudo 2 e Estudo 3 que, no seu conjunto, permitiram a análise e discussão relativamente às práticas de enfermagem centradas na família.

\section{Estudo 2 - Cuidar a Família: dos conceitos às representações}

Adoptamos como referencial teórico a Teoria das Representações Sociais. As representações contribuem para a construção de uma realidade comum a um conjunto social, constituídas por crenças e valores dos grupos que, através da comunicação orientam a produção do sujeito social. Sendo atributos de grupo, as representações funcionam como quadro de referência, tornando previsível o comportamento dos indivíduos ou do grupo ${ }^{(17)}$.
No contexto da fase de diagnóstico e de acordo com o referencial teórico, definimos os seguintes objectivos: Identificar os elementos das representações dos enfermeiros sobre Família, Enfermagem de Família e Saúde Familiar e descrever a forma como estes se organizam.

A população do estudo foi constituída pelos enfermeiros de família do contexto onde desenvolvemos a pesquisa, no total de 22 enfermeiros.

Os dados foram recolhidos através da auto-administração de um questionário, durante os meses de Maio e Junho de 2005, sendo os dados submetidos à análise de conteúdo estrutural. A análise foi efectuada a partir de um quadro teórico de base em que as dimensões e o sistema de categorias resultaram da classificação analógica e progressiva dos elementos.

Os resultados evidenciaram que nas representações de Família emergem duas perspectivas no sistema de pensamento dos enfermeiros: sociológica e psicológica, sugerindo-nos cognições associadas ao conceito da Classificação Internacional para a Prática de Enfermagem (CIPE) ${ }^{(4)}$. Relativamente às representações de Saúde Familiar, emergem cognições ligadas respectivamente às categorias bem-estar, psicológico e grupo familiar, que nos parecem ser baseadas no conceito de Saúde Individual. As representações de Enfermagem de Família encontram-se estruturadas em três dimensões: níveis de intervenção, caracterização e objectivos da intervenção, salientando-se a primeira como mais relevante, sendo as evocações dos enfermeiros distribuídas pelos quatro níveis de intervenção ${ }^{(11)}$, com maior preponderância na família como contexto. A caracterização da intervenção surge associada aos domínios desenvolvimental e funcional da família, com representações direccionadas para o promover, manter e restaurar a saúde da família.

\section{Estudo 3 - Práticas centradas na família: descrição dos cuidados}

Para completar a análise dos modelos em uso ${ }^{(16)}$, norteamo-nos pelos seguintes objectivos: identificar os tipos de famílias sujeitas à intervenção dos enfermeiros; identificar os focos de atenção utilizados na avaliação inicial da família e nos enunciados diagnósticos; identificar as intervenções de enfermagem dirigidas às famílias e as estratégias de avaliação dos resultados; analisar os modelos conceptuais da prática de cuidados à família e analisar a percepção dos enfermeiros sobre os obstáculos à prática de enfermagem de família.

A recolha de dados processou-se através da auto-administração de um questionário aos 22 enfermeiros de família do contexto de estudo e da análise da documentação produzida pelos mesmos enfermeiros no SAPE, no decorrer dos meses de Maio e Junho de 2005. 
O tratamento e análise dos dados foi realizado através de técnicas quantitativas utilizando-se análise estatística descritiva e testes não paramétricos associadas a técnicas qualitativas como a análise de conteúdo. Para a efectivação desta análise tivemos como referenciais o Modelo Calgary de Avaliação da Família ${ }^{(1)}$ e o Modelo Calgary de Intervenção na Família ${ }^{(1)}$. Articulamos os conceitos dos mesmos com a Classificação Internacional para a Prática de Enfermagem (CIPE), versão Beta2, visto os padrões de documentação do Sistema de Informação de Enfermagem em uso, serem baseados nesta versão.

Na complexidade dos resultados obtidos, salientamos aqueles que nos parecem mais relevantes na compreensão da intervenção familiar, neste contexto de cuidados:

- As famílias, em todas as etapas do seu ciclo vital foram consideradas como alvo da intervenção dos enfermeiros;

- Divergência entre a descrição dos focos mais valorizados na avaliação familiar e a documentação dos dados de avaliação inicial da família. Estas divergências salientaramse em todas as dimensões avaliativas: estrutural, desenvolvimental e funcional;

- Focos de atenção valorizados pelos enfermeiros e utilizados nos enunciados diagnósticos, mas sem a descrição dos dados que permitam a fundamentação do diagnóstico;

- Descrição das práticas não direccionadas à família como unidade e alvo dos cuidados;

- Concepção de cuidados não fundamentada em modelos conceptuais que permitam a organização dos fenómenos numa abordagem sistémica da família.

- Os aspectos percepcionados pela equipa como impeditivos da prática de enfermagem de família, são direccionados para as próprias famílias (não estão preparadas para uma intervenção diferenciada), a formação (formação escassa na área de enfermagem de família), os modelos da prática (inexistência de modelos orientadores) e aspectos organizacionais (recursos e organização dos cuidados).

Após o desenvolvimento dos três estudos que englobam a fase diagnóstica do ciclo de investigação acção, foram realizadas várias reuniões com a equipa de enfermagem, visando a confrontação, discussão e reflexão sobre os resultados obtidos.

Foi consensual a necessidade de alterar o modelo em uso dos cuidados de enfermagem direccionados à família, aproximando-o dos modelos conceptuais e formativos.

Além dos aspectos considerados pela equipa como factores impeditivos da concretização de práticas centradas na família, os enfermeiros manifestaram dificuldades na definição das situações de cuidados em que a família é o alvo, na actividade diagnóstica, na compreensão sistémica da família e na utilização do SAPE, por considerarem que os padrões de documentação não permitem o registo de uma avaliação sistemática e global das famílias.
A partir destes processos de discussão e reflexão foi possível iniciarmos a fase seguinte, de definição de estratégias para a implementação da mudança.

\section{Fase de planeamento da aç̧ão}

Considerando a análise global dos dados e as estratégias discutidas com a equipa, foi elaborado o plano relativo a esta fase e validado com os orgãos de gestão da instituição, integrando duas etapas principais: Processo Formativo e Redefinição do Modelo de Cuidados em Uso.

\section{1 - Processo Formativo}

Para o processo formativo foram definidos objectivos conducentes ao desenvolvimento de competências que permitissem a concepção da família tendo por base o paradigma sistémico e, desta forma, possibilitasse a redefinição do modelo de cuidados.

Os conteúdos direccionaram-se para aspectos essenciais dos cuidados às famílias, introduzindo-se conteúdos no âmbito dos Cuidados de Saúde Primários, dos Sistemas de Informação em Enfermagem e da CIPE.

Este dispositivo foi desenvolvido no Centro de Saúde onde decorreu a investigação, formando-se quatro grupos de formação/discussão, com a totalidade de 80 horas formativas, no período de Julho a Dezembro de 2006. Ao longo da formação foram introduzidas temáticas que emergiam da discussão e mobilizados recursos formativos no sentido de dinamizar este processo.

\section{2 - Redefinição do Modelo de Cuidados em Uso}

Para a concretização desta actividade, desenvolvemos as seguintes etapas:

- (Re)Construção do Modelo de Cuidados de Enfermagem à Família tendo como referenciais o Pensamento Sistémico $^{(9)}$, o Modelo Calgary de Avaliação da Família (MCAF) ${ }^{(1)} \mathrm{e}$ o Modelo Calgary de Intervenção na Família (MCIF) ${ }^{(1)}$.

- Operacionalização do Modelo através da definição de: dados para avaliação familiar; àreas de atenção com relevância para a prática de enfermagem de família; sub-conjuntos diagnósticos,intervenções e resultados de enfermagem direccionados para a Enfermagem de Família.

- Plano de adequação dos padrões de documentação à estrutura do Modelo de Cuidados (Re)Construído.

Para a concretização destas actividades utilizamos a técnica de debates de grupo ${ }^{(18)}$, com debates semanais de subgrupos, debates mensais do grupo geral e ainda discussões temáticas por pequenos grupos.

Conscientes da necessidade de envolver todos os enfermeiros na tomada de decisão, tentou-se dinamizar de forma criativa os debates, mobilizando o conhecimento prático dos intervenientes para os processos de mudança. 
$\mathrm{Na}(\mathrm{Re})$ Construção do Modelo de Cuidados, além da análise global da inter-relação dos conceitos do $\mathrm{MCAF}^{(1)} \mathrm{e}$ dos constructos associados às categorias do modelo, discutiram-se situações reais de cuidados à família que permitiram ampliar a visão de família e dos fenomenos de compreensão da mesma.
Os dados de avaliação familiar são consentâneos à discussão do modelo, e permitiram a sistematização de dados diagnósticos associados às areas de atenção, consideradas relevantes na prática de enfermagem de família. A partir destes pressupostos e, em articulação com a CIPE, foram definidos as areas de atenção descritas a seguir (Figura 1).

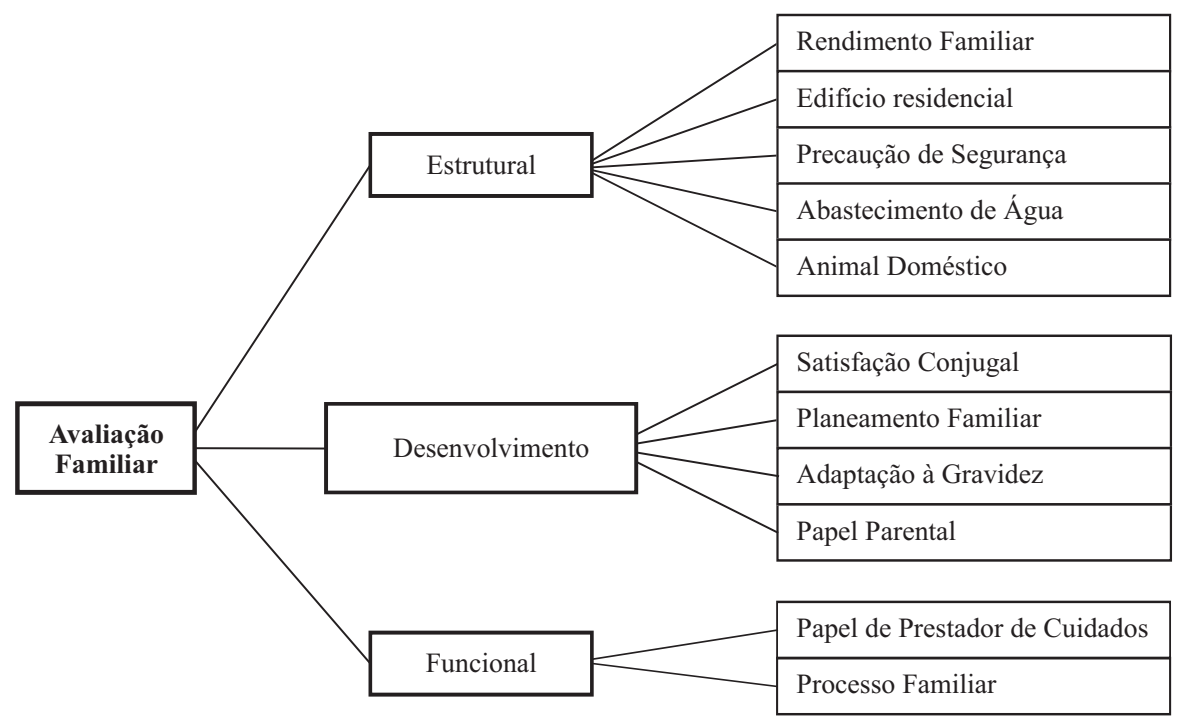

Figura 1- Diagrama do Modelo de Cuidados de Enfermagem de Família (Reconstruído)

Pretendendo que este seja um modelo dinâmico e interactivo, definimos, a partir das áreas de atenção, os subconjuntos diagnósticos e diagnósticos que, com a família, e através da identificação das suas forças e recursos, nos permitem esboçar um plano de cuidados adequado.

Para a definição dos sub-diagnósticos e diagnósticos, foi necessário englobar dimensões avaliativas para cada uma das áreas de atenção. Designamos como dimensões outros focos da prática de enfermagem ${ }^{(4)}$, que, de acordo com as discussões que emergiram nos debates de grupo, foram considerados como componentes do foco central. Por exemplo, relativamente à área de atenção Processo Familiar, designado como foco central, foram agregados outros focos, considerados como dimensões avaliativas do mesmo: Comunicação Familiar; Coping Familiar; Interação de Papéis; Relação Dinâmica. A opinião clínica do enfermeiro, relativamente a cada uma destas dimensões, será considerado como um subdiagnóstico, enquanto a sua decisão relativamente ao foco central, Processo Familiar, um diagnóstico.

Esta metodologia foi utilizada para a formulação de todos os enunciados diagnósticos, a partir das áreas de atenção consideradas relevantes na prática dos cuidados centrados na família como unidade de intervenção, descritas anteriormente.

A última etapa da operacionalização do modelo, correspondeu à construção dos enunciados das intervenções para cada um dos sub-diagnósticos e diagnósticos centrais. Para a concretização desta etapa tivemos como referencial o $\mathrm{MCIF}^{(1)}$, que se constituiu como base para reflexão. Paulatinamente, de acordo com as idéias que iam emergindo dos debates, introduzimos conceitos e estratégias de outros modelos de intervenção familiar, sobretudo da Terapia Familiar, que permitiram a reflexão das práticas com base na evidência.

Finalizada a etapa de reconstrução e operacionalização do Modelo de Cuidados, manteve-se os debates de grupo, que decorreram de Janeiro a Novembro de 2007, no sentido de estabelecer padrões de documentação que fossem congruentes com o Modelo e simultaneamente passíveis de serem utilizados no Sistema de Informação em uso. Estruturamse todos os dados obtidos por áreas de atenção, sistematizados a partir das áreas inicias de avaliação familiar.

\section{IMPLEMENTAÇÃO}

O SAPE, Sistema de Informação em Enfermagem, é constituído por software que processa informação e permite o acesso dos profissionais para consulta e posterior análise.

A alteração das especificações da interface gráfica do mesmo sistema, foi autorizada pelos órgãos de gestão da Instituição e também pela Administração Central do Sistema de Saúde (ACSS), que é responsavel pela gestão dos sistemas e tecnologias de informação do Serviço Nacional de Saúde.

Para a efectiva transformação das práticas dos cuidados e de acordo com o plano de preparação para a mudança, no decorrer do mês de Dezembro de 2007, procedemos às alterações na aplicação informática, estruturando os dados de acordo com o Modelo (Co)Construído. 
Com a realização desta alteração de mudança efectiva no contexto da prática, e com o envolvimento constante de todos os intervenientes nos processo anteriores, julgamos existirem condições para que, de um paradigma e prática de cuidados centrados no indivíduo, se passe para uma prática centrada na família como cliente, numa abordagem sistémica da mesma.

\section{CONSIDERAÇÕES FINAIS}

Para a avaliação da mudança das práticas, recorremos ao modelo de análise que foi utilizado na fase de diagnóstico, no que se referiu à identificação do modelo em uso. Pretendemos comparar as representações, interpretação e descrição dos cuidados de enfermagem, identificando as mudanças produzidas a estes três níveis. Com a concretização desta avaliação será então possível a identificação do conhecimento prático produzido concomitantemente à produção da teoria.

A (co)construção do modelo a partir das necessidades identificadas pelos enfermeiros, legitimado pelas tomadas de decisão dos mesmos, constitui-se, de alguma forma, como um processo de discussão teórico e prático em enfermagem de família. Discutindo com base num referencial de abordagem sistémica, emergindo constantemente a multidimensionalidade e complexidade inerentes aos cuidados

\section{REFERÊNCIAS}

1. Wright LM, Leahey M. Enfermeiras e famílias: um guia para a avaliação e intervenção na família. São Paulo: Roca; 2002.

2. Hanson SM. Enfermagem de cuidados de saúde à família: teoria, prática e investigação. Camarate: Lusociência; 2005.

3. Wall K. Famílias no Censo 2001: estruturas domésticas em Portugal. Sociologia. 2003;43(1):9-11.

4. Conselho Internacional de Enfermeiros (ICN). Classificação Internacional para a Prática de Enfermagem - CIPE. versão beta 2. Lisboa: IGIF/APE; 2002.

5. Minuchin S. Famílias: funcionamento e tratamento. Porto Alegre: Artes Médicas; 1990.

6. Bertalanffy V. Teoria geral dos sistemas. Petrópolis: Vozes; 1977.

7. Wiener N. Cybernetics: or control and communication in the animal and the machine. Massachusetts: Massachusetts Institute of Technology; 1948.

8. Watzlawick P, Beavin JH, Jackson D. Pragmática da comunicação humana. São Paulo: Cultrix; 1967.

9. Vasconcellos MJ. Pensamento sistêmico: o novo paradigma da ciência. São Paulo: Papirus; 2002.

10. Whyte D. Explorations in family nursing. London : Routledge; 1977. à família, julgamos que a definição das áreas de intervenção permitirá a implementação de estratégias conducentes à produção de resultados.

A operacionalização do Modelo teve como base os pressupostos do processo de enfermagem, através dos elementos: diagnósticos, intervenção e resultados. A sistematização dinâmica destes elementos ajudam a determinar o que deve ser feito, porque deve ser feito, por quem deve ser feito; como deve ser feito e que resultados são esperados com a execução das intervenções de enfermagem. As intervenções implementadas terão sempre como finalidade capacitar a família para a mudança nos seus três domínios de funcionamento: cognitivo, afectivo e comportamental ${ }^{(1)}$.

Incluir os sistemas de informação no percurso de pesquisa, partiu de uma necessidade expressa pelos enfermeiros, sendo cada vez mais real a sua influência nas práticas pela quantidade e complexidade da informação. Acrescenta-se ainda o facto de que, a documentação dos cuidados favorecem, o planeamento de actividades e asseguram a continuidade dos cuidados de enfermagem. Por isso concebemos um modelo conceptual de cuidados à família e estruturamos os dados no sistema com base no mesmo. Cremos que, com a implementação dos processos de mudança, se tornará possível dar visibilidade aos contributos dos cuidados de enfermagem no contexto dos cuidados direccionados à família como unidade de intervenção.

11. Friedman MM. Family nursing research, theory and practice. Stamford: Appleton \& Lange; 1998.

12. Gilliss $C L$, Highley $B L$, Roberts $B M$, Martinson $M$, editors. Toward a science of family nursing. New York: Addson-Wesley; 1989.

13. Martins MM. Uma crise acidental na família: o doente com AVC. Coimbra: FORMASAU; 2002.

14. Püschel VA, Ide CA, Chaves EC. Modelos clínicos e psicossociais de atenção ao indivíduo e à família na assistência domiciliar: bases conceituais. Rev Esc Enferm USP. 2006;40(2): 261-8.

15. Streubert HJ, Carpenter DR. Investigação qualitativa em enfermagem: avançando o imperativo humanista. Camarate: Lusociência; 2002.

16. Silva A. Sistemas de Informação em Enfermagem: uma teoria explicativa da mudança. Coimbra: FORMASAU; 2006.

17. Moscovici S. La psychanalyse, son image et son publique. Paris: PUF; 1976.

18. Flick U. Métodos qualitativos na investigação científica. Lisboa: Monitor; 2005. 